\title{
SB@GU at the Complex Word Identification 2018 Shared Task
}

\author{
David Alfter \\ Språkbanken \\ University of Gothenburg \\ Sweden \\ david.alfter@gu.se
}

\author{
Ildikó Pilán \\ Språkbanken \\ University of Gothenburg \\ Sweden \\ ildiko.pilanegu.se
}

\begin{abstract}
In this paper, we describe our experiments for the Shared Task on Complex Word Identification (CWI) 2018 (Yimam et al., 2018), hosted by the $13^{\text {th }}$ Workshop on Innovative Use of NLP for Building Educational Applications (BEA) at NAACL 2018. Our system for English builds on previous work for Swedish concerning the classification of words into proficiency levels. We investigate different features for English and compare their usefulness using feature selection methods. For the German, Spanish and French data we use simple systems based on character n-gram models and show that sometimes simple models achieve comparable results to fully featureengineered systems.
\end{abstract}

\section{Introduction}

The task of identifying complex words consists of automatically detecting lexical items that might be hard to understand for a certain audience. Once identified, text simplification systems can substitute these complex words by simpler equivalents to increase the comprehensibility (readability) of a text. Readable texts can facilitate information processing for language learners and people with reading difficulties (Vajjala and Meurers, 2014; Heimann Mühlenbock, 2013; Yaneva et al., 2016).

Building on previous work for classifying Swedish words into different language proficiency levels (Alfter and Volodina, 2018), we extend our pipeline with English resources. We explore a large number of features for English based on, among others, length information, parts of speech, word embeddings and language model probabilities. In contrast to this feature-engineered approach, we use a word-length and n-gram probability based approach for the German, Spanish and French data.
Our interest for participation in this shared task is connected to the ongoing development of a complexity prediction system for Swedish (Alfter and Volodina, 2018). In contrast to this shared task, we perform a five-way classification corresponding to the first five levels of the CEFR scale of language proficiency (Council of Europe, 2001). We adapted the pipeline to English, and included some freely available English resources to see how well these would perform on the CWI 2018 task and to gain insights into how we could improve our own system.

\section{Data}

There were four different tracks at the shared task. Table 1 shows the number of annotated instances per language. For the French sub-task, no training data was provided. Each instance in the English dataset was annotated by 10 native speakers and 10 non-native speakers. For the other languages, 10 annotators (native and non-native speakers) annotated the data. An item is considered complex if at least one annotator annotates the item as complex.

\begin{tabular}{lll}
\hline Language & Training & Development \\
\hline English & 27299 & 3328 \\
Spanish & 13750 & 1622 \\
German & 6151 & 795 \\
French & $/$ & $/$ \\
\hline
\end{tabular}

Table 1: Number of instances per language

In the dataset, information about the total number of native and non-native annotators and how many of each category considered a word complex is also available.

A surprising aspect of the 2018 dataset was the presence of multi-word expressions (MWE), which were not part of the 2016 shared task. For 
the 2018 task, the training data contains $14 \%$ of MWEs while the development data contains $13 \%$.

\section{Features}

We extract a number of features from each target item, either a single word or a multi-word expression. The features can be grouped into: (i) count and word form based features, (ii) morphological features, (iii) semantic features and (iv) context features. In addition, we use psycholinguistic features extracted by N-Watch (Davis, 2005). In Table 2, we list the complete set of features used for English.

\begin{tabular}{l}
\hline Count features \\
\hline Length (number of characters) \\
Syllable count (S1) \\
Contains non-alphanumeric character \\
Is number \\
Is MWE \\
Character bigrams (B1) \\
N-gram probabilities (Wikipedia) \\
In Ogden list \\
AWL distribution \\
CEFRLex distribution \\
\hline Morphological features \\
\hline Part-of-speech \\
Suffix length \\
\hline Semantic features \\
\hline Number of synsets \\
Number of hypernyms \\
Number of hyponyms \\
Sense id \\
\hline Context features \\
\hline Topic distributions \\
Word embeddings \\
\hline N-Watch features \\
\hline British National Corpus frequency (BNC) \\
CELEX frequency (total, written, spoken) \\
In Kučera Francis (KF) list \\
Sydney Morning Herald frequency (SMH) \\
Reaction time \\
Bigram frequency (B2) \\
Trigram frequency (T2) \\
Syllable count (S2) \\
\hline
\end{tabular}

Table 2: Overview of features
Word length in terms of number of characters has been shown to correlate well with complexity in a number of studies (Smith, 1961; Björnsson, 1968; O'Regan and Jacobs, 1992).

Besides the number of characters, we also consider the number of syllables (S1 and S2). As the calculation of syllables in English is not straightforward, we use a lookup-based method for S1. In case the word is not present in the lookup list, we apply a heuristic approach as a fall-back. A high number of multi-syllabic words has been shown to increase the overall complexity of a text (Flesch, 1948; Kincaid et al., 1975), so we assume it could also be helpful in predicting the complexity of smaller units.

The feature related to bigrams (B1) indicates which character bigrams occur in the target item. We calculate all character-level bigrams in the training data and only retain the 36 most predictive bigrams using Correlation-based Feature Subset Selection (Hall, 1999).

$\mathrm{N}$-gram probabilities are based on language models trained on the English Wikipedia dumps from June and July $2015^{1}$. We calculate characterlevel unigram, bigram and trigram probabilities.

The Ogden list contains 850 words from Basic English (Ogden, 1944) and this feature indicates whether a word is part of this list.

AWL distribution considers the ten Academic Word List (AWL) sublists (Coxhead, 1998) and indicates in which lists the word occurs. The AWL list contains word families which appear often in academic texts but excludes general English vocabulary, making it specific to the academic context. The ten sub-lists are ordered according to frequency, so that words from the first sub-list are more frequent than words from the second sublist, and so forth.

CEFRLex distribution indicates the presence/absence in the $5^{\text {th }}, 10^{\text {th }}$ and $20^{\text {th }}$ percentile English CEFRLex lists ${ }^{2}$. These lists are obtained by aligning and sorting four different vocabulary lists for English (EFLLex) (Dürlich and François, 2018), French (FLELex) (François et al., 2014), Swedish (SVALex) (François et al., 2016) and Dutch (NT2Lex) (François and Fairon, 2017) by frequency and only taking words which occur in

\footnotetext{
${ }^{1}$ We already had these pre-calculated language models from previous experiments. For simplicity and time reasons, we chose not to retrain them on more recent Wikipedia dumps.

${ }^{2}$ http://cental.uclouvain.be/cefrlex/
} 
the $5^{\text {th }}, 10^{\text {th }}$ and $20^{\text {th }}$ percentile across all languages.

Morphological features include information about parts of speech and suffix length. Suffix length is calculated by stemming the word using the NLTK stemmer (Bird et al., 2009) and substracting the length of the identified stem from the length of the original word.

Semantic features are: number of synsets, number of hyponyms, number of hypernyms and sense id. These features are calculated from WordNet (Miller and Fellbaum, 1998). The first three are obtained by calculating how many items WordNet returns for the word in terms of synsets, hyponyms and hypernyms. Sense id is obtained by using the Lesk algorithm (Lesk, 1986) on the sentence the target item occurs in.

Context features consist of topic distribution and word embeddings. For word embeddings, we use the pre-trained Google News dataset embeddings. We calculate the word context of a word $w_{i}$ in a sentence $S \in w_{1} \ldots w_{n}$ as the sum of word vectors from $w_{i-5}$ to $w_{i+5}$, excluding the vector for $w_{i}$. In case there is not enough context, the available context is used instead. Topic distributions are calculated by first collecting Wikipedia texts about 26 different topics such as animals, arts, education or politics. These texts are tokenized and lemmatized. We then exclude words which occur across all topic lists. Topic distribution indicates in which of these topic lists the target item occurs.

Features from N-Watch include frequency information from the British National Corpus (BNC), the English part of CELEX, the Kučera and Francis list (KF), the Sydney Morning Herald $(\mathrm{SMH})$; reaction times and bi- and trigram character frequencies (B2 and T2). While these features are redundant in some case, such as number of syllables (S1 and S2), their values can differ due to being calculated differently.

Since our pipeline was not designed to handle multi-word expressions, we address this by a twopass approach. First, we extract all features for single words and store the resulting vector representations. Then, for each multi-word expression, if we have feature vectors for all constituents making up the MWE, we sum the vectors for countbased features such as length and number of syllables and average the vectors for frequency counts. We have experimented with adding all vectors and averaging all vectors, but found that summing some features and averaging other features not only yields higher scores but also is linguistically more plausible. Context vectors for MWEs are not added but calculated separately as described above with the difference that for a multi-word expression MWE $\in w_{i}, \ldots, w_{i+k}$ occurring in a sentence $S \in w_{1}, \ldots, w_{n}$ as the sum of vectors from $w_{i-5}$ to $w_{i-1}$ and $w_{i+k+1}$ to $w_{i+k+5}$. In case not all constituents of a multi-word expression have corresponding vectors from phase 1 , we set all feature values to zero and only use the context.

\section{Experiments on the English data}

We tried three different configurations for the English data set, namely context-free, context-only and context-sensitive. For context-free, we use the features described above, excluding word embedding context. For context-only, we only use the word embedding context vectors.For contextsensitive, we concatenate the context-free and context-only features.

\subsection{Classification}

We tried different classifiers, among others Random Forest (Breiman, 2001), Extra Trees (Geurts et al., 2006), convolutional neural networks and recurrent convolutional neural networks implemented in Keras (Chollet et al., 2015) and PyTorch (Paszke et al., 2017). For Random Forest and Extra Trees, we tried different numbers of estimators in the interval $[10,2000]$ and found that generally either 500 or 1000 estimators reached the best results on the development set. For neural networks, we tried different combinations of hyperparameters such as the type of layers, number of convolution filters, adding LSTM layers, varying the number of neurons in each layer. We tried two different architectures, one taking as input the features extracted as described below and convolving over these features, the other taking both the features and word embeddings as separate inputs and merging the separate layers before the final layer.

\section{Experiments on other languages}

\subsection{Predicting the German and the Spanish test set}

During testing, we noticed that using the character-level n-gram model trained on the English Wikipedia and using only unigram, bigram and trigram probabilities and word length as features yielded scores in the vicinity of our best- 
performing feature-engineered models at that time (0.81 F1 vs $0.82 \mathrm{~F} 1$ ).

Following this finding, we used character-level n-gram models trained on Wikipedia dumps ${ }^{3}$ for Spanish, German and French and calculated unigram, bigram and trigram probabilities for these languages. In addition, we used target item length in characters as additional feature.

\subsection{Predicting the French test set}

As there was no training or development data for the French test set, we used the n-gram language model to convert each French entry into n-gram probabilities. We then used the n-gram classifiers for English, German and Spanish to predict labels for each word. We tested two configurations:

1. Predict with English, German and Spanish classifier and use majority vote to get the final label

2. Predict with Spanish classifier and use label as final label

The rationale behind the second configuration is that French and Spanish are both Romance languages. The single Spanish classifier might thus model French data better than incorporating also the English and the German classifiers, as German and English are both Germanic languages.

\section{Results}

Table 3 shows the results of the best classifiers on both the development data and the test data. For the English News and WikiNews, the best classifier is an Extra Trees classifier with 1000 estimators with the reduced feature set (see subsection 6.1) and trained on each genre separately, as opposed to the general English classifier trained on all three genres. For all other tasks, the best classifier is an Extra Trees classifier with 500 estimators with the reduced feature set.

\subsection{Feature selection for English}

Out of the set of features proposed for a certain task, usually some features are more useful than others. Eliminating redundant features can result not only in simpler models, but it can also improve performance (Witten et al., 2011, 308). We

\footnotetext{
${ }^{3}$ See footnote 1
}

\begin{tabular}{lll}
\hline & F1 (dev) & F1 (test) \\
\hline EN News & 0.8623 & 0.8325 \\
EN WikiNews & 0.8199 & 0.8031 \\
EN Wikipedia & 0.7666 & 0.7812 \\
German & 0.7668 & 0.7427 \\
Spanish & 0.7261 & 0.7281 \\
French & $/$ & 0.6266 \\
\hline
\end{tabular}

Table 3: Results of best classifiers

therefore run feature selection experiments in order to identify the best performing subset of features. We use the SelectFromModel ${ }^{4}$ feature selection method as implemented in scikit-learn (Pedregosa et al., 2011). This method selects features based on their importance weights learned by a certain estimator. We base our selection on the development data and the Extra Trees learning algorithm, since it performed best with the full set of features. We use the median of importances as threshold for retaining features. For the other parameters, the default values were maintained for the selection.

The feature selection method identified a subset of 64 informative features. We list these features in Table 4, indicating in parenthesis the amount of features per feature type where it is relevant.

\begin{tabular}{ll}
\hline \multicolumn{2}{c}{ Selected features } \\
\hline Length & Sense id \\
Is adjective & \# Syllable count S2 \\
Is noun & BNC freq. \\
Is verb & CELEX freq. (3) \\
Syllable count S1 & KF list \\
Suffix length & Reaction time \\
\# synsets & SMH \\
\# hypernyms & Bigram B2 freq (4) \\
\# hyponyms & Trigram T2 freq (4) \\
Topic distr. (22) & Is MWE \\
Char. bigram B1 (8) & Unigram prob \\
In Ogden list & Bigram prob \\
CEFRLex distr. (3) & Trigram prob \\
\hline
\end{tabular}

Table 4: Selected subset of features

The best performing features included, among others, features based on word frequency, infor-

\footnotetext{
${ }^{4}$ We also tested other feature selection methods, namely an ANOVA-based univariate feature selection and recursive feature elimination, but we omit the results of these since they were inferior.
} 
mation based on words senses and topics as well as language model probabilities.

As only lexical classes were annotated for complexity, it is not surprising to see that, even though our pipeline considers all part-of-speech classes, the feature selection picked adjectives, nouns and verbs.

\section{Additional experiments on English}

\subsection{Native vs non-native}

Since we had information about how many native speakers and non-native speakers rated target items as complex, we experimented with training classifiers separately for these two categories of raters. We applied the native-only classifier on the native judgments of the development set, as well as on the non-native judgments, and similarly the non-native classifier on native judgments and nonnative judgments. In all four configurations, we found accuracy to be the same, at about $75 \%$.

\section{$7.2 \quad 2016$ vs 2018}

Before this shared task, we experimented with the 2016 CWI shared task data and trained classifiers on it. We tried applying the best-performing classifier trained on the 2016 data on the 2018 development data, but results were inferior to training on the 2018 training data and predicting 2018 development data. The same is true in the other direction; applying the best-performing 2018 classifier on the 2016 data yields inferior results. Table 5 shows the result of these experiments. This raises the question of how generalizable these complex word identification systems are and how dependent they are on the data, the annotation and the task at hand.

\begin{tabular}{llll}
\hline Configuration & \multicolumn{2}{l}{ Accuracy Recall } & F1 \\
\hline 2016 on 2018 & 0.6499 & 0.7463 & 0.6948 \\
2018 on 2018 & 0.7992 & 0.7269 & 0.7613 \\
\hline 2018 on 2016 & 0.6610 & 0.6335 & 0.6470 \\
2016 on 2016 & 0.8062 & 0.6511 & 0.7204 \\
\hline
\end{tabular}

Table 5: Results of 2016/2018 comparison

\subsection{Genre dependency}

During the training phase, we concatenated the English training files for News, WikiNews and Wikipedia into one single training file. We did the same with the development data. We trained a single, genre-agnostic English classifier on this data. During the submission phase, we used the single classifier but also split the data into the three sub-genres News, WikiNews and Wikipedia again and retrained our systems, which improved performance. This hints at the genre-dependency of the concept of complex words.

\subsection{Context}

As the notion of complexity may be contextdependent, i.e. a word might be perceived as more complex in a certain context, we used word embedding context vectors as features. However, our feature selection methods show that these context vectors do not contribute much to the overall classification results. Indeed, of the 300-dimensional word embedding vectors representing word context, not a single dimension was selected by our feature selection.

However, if we only look at features which can be derived from isolated words, we also have a problem of contradictory annotations. This means that representing isolated words as vectors can lead to the same vector representation of different instances of a word with different target labels. We calculated the number of contradictions and found that representing each word as a vector leads to $5 \%$ of contradictory data points.

\section{Discussion}

One interesting aspect of the data is the separation of annotators into native and non-native speakers. However, while it can be interesting to try and train separate classifiers for modeling native and non-native perceptions of complexity, and this information can be exploited at training time, using features that rely on the number of native and non-native annotators could not be used on the test data, as the only information given at test time is the total number of native and non-native annotators, and these numbers do not vary for the English data.

Our best classifiers are all Extra Trees. All other classifiers that we tested, especially convolutional neural networks and recurrent convolutional neural networks, reached lower accuracies. This might be due to insufficient data to train neural networks, a suboptimal choice of hyperparameters or the type of features used.

While our systems did not reach high ranks on the English datasets (ranks 13, 13 and 6 on 
News, WikiNews and Wikipedia respectively), we reached place 2 on the German data set and place 3 on the French data set. Given the simplicity of the chosen approach, this is slightly surprising. However, we surmise that n-gram probabilities implicitly encode frequency among other things, and frequency-based approaches generally perform well.

Further, we found that using only the Spanish classifier on the French data lead to better scores than using all three classifiers and majority vote. This speaks in favor of the hypothesis that closely related languages model each other better. This can be interesting for low-resource languages if there is a related language with more resources.

\section{Conclusion}

We presented our systems and results of the 2018 shared task on complex word identification. We found that simple $\mathrm{n}$-gram language models perform similarly well to fully-feature engineered systems for English. Our submission for the nonEnglish tracks were based on this observation, circumventing the need for more language-specific feature engineering.

\section{Acknowledgements}

We would like to thank our anonymous reviewer for their helpful comments and the organizers of the shared task for the opportunity to work on this problem.

\section{References}

David Alfter and Elena Volodina. 2018. Towards Single Word Lexical Complexity Prediction. In Proceedings of the 13th Workshop on Innovative Use of NLP for Building Educational Applications.

Steven Bird, Ewan Klein, and Edward Loper. 2009. Natural language processing with Python: analyzing text with the natural language toolkit. O'Reilly Media, Inc.

Carl Hugo Björnsson. 1968. Läsbarhet. Liber.

Leo Breiman. 2001. Random forests. Machine Learning, 45(1):5-32.

François Chollet et al. 2015. Keras. https:// keras.io.

Council of Europe. 2001. Common European Framework of Reference for Languages: Learning, Teaching, Assessment. Press Syndicate of the University of Cambridge.
Averil Coxhead. 1998. An academic word list, volume 18. School of Linguistics and Applied Language Studies.

Colin J Davis. 2005. N-Watch: A program for deriving neighborhood size and other psycholinguistic statistics. Behavior research methods, 37(1):65-70.

Luise Dürlich and Thomas François. 2018. EFLLex: A Graded Lexical Resource for Learners of English as a Foreign Language. In 11th International Conference on Language Resources and Evaluation (LREC 2018).

Rudolph Flesch. 1948. A new readability yardstick. Journal of applied psychology, 32(3):221.

Thomas François and Cédrick Fairon. 2017. Introducing NT2Lex: A Machine-readable CEFR-graded Lexical Resource for Dutch as a Foreign Language. In Computational Linguistics in the Netherlands 27 (CLIN27).

Thomas François, Nuria Gala, Patrick Watrin, and Cédrick Fairon. 2014. FLELex: a graded lexical resource for French foreign learners. In $L R E C$, pages 3766-3773. Citeseer.

Thomas François, Elena Volodina, Ildikó Pilán, and Anaïs Tack. 2016. SVALex: a CEFR-graded Lexical Resource for Swedish Foreign and Second Language Learners. In LREC.

Pierre Geurts, Damien Ernst, and Louis Wehenkel. 2006. Extremely randomized trees. Machine Learning, 63(1):3-42.

Mark Andrew Hall. 1999. Correlation-based feature selection for machine learning.

Katarina Heimann Mühlenbock. 2013. I see what you meanAssessing readability for specific target groups. Data linguistica, (24).

J Peter Kincaid, Robert P Fishburne Jr, Richard L Rogers, and Brad S Chissom. 1975. Derivation of new readability formulas (automated readability index, fog count and flesch reading ease formula) for navy enlisted personnel. Technical report, Naval Technical Training Command Millington TN Research Branch.

Michael Lesk. 1986. Automatic sense disambiguation using machine readable dictionaries: how to tell a pine cone from an ice cream cone. In Proceedings of the 5th annual international conference on Systems documentation, pages 24-26. ACM.

George Miller and Christiane Fellbaum. 1998. Wordnet: An electronic lexical database.

Charles Kay Ogden. 1944. Basic English: A general introduction with rules and grammar, volume 29 . K. Paul, Trench, Trubner. 
J Kevin O'Regan and Arthur M Jacobs. 1992. Optimal viewing position effect in word recognition: A challenge to current theory. Journal of Experimental Psychology: Human Perception and Performance, 18(1):185

Adam Paszke, Sam Gross, Soumith Chintala, Gregory Chanan, Edward Yang, Zachary DeVito, Zeming Lin, Alban Desmaison, Luca Antiga, and Adam Lerer. 2017. Automatic differentiation in PyTorch. In NIPS-W.

Fabian Pedregosa, Gaël Varoquaux, Alexandre Gramfort, Vincent Michel, Bertrand Thirion, Olivier Grisel, Mathieu Blondel, Peter Prettenhofer, Ron Weiss, Vincent Dubourg, et al. 2011. Scikit-learn: Machine learning in Python. Journal of Machine Learning Research, 12(Oct):2825-2830.

Edgar A Smith. 1961. Devereux Readability Index. The Journal of Educational Research, 54(8):298303.

Sowmya Vajjala and Detmar Meurers. 2014. Assessing the relative reading level of sentence pairs for text simplification. In Proceedings of the $14^{\text {th }}$ Conference of the European Chapter of the Association for Computational Linguistics.

Ian H Witten, Eibe Frank, Mark A Hall, and Christopher J Pal. 2011. Data Mining: Practical machine learning tools and techniques. Morgan Kaufmann.

Victoria Yaneva, Irina P Temnikova, and Ruslan Mitkov. 2016. Evaluating the Readability of Text Simplification Output for Readers with Cognitive Disabilities. In LREC.

Seid Muhie Yimam, Chris Biemann, Shervin Malmasi, Gustavo Paetzold, Lucia Specia, Sanja Štajner, Anaïs Tack, and Marcos Zampieri. 2018. A Report on the Complex Word Identification Shared Task 2018. In Proceedings of the 13th Workshop on Innovative Use of NLP for Building Educational Applications, New Orleans, United States. Association for Computational Linguistics. 